\title{
Hyperspectral chemical agent standoff detection using sparse representation (Withdrawal Notice)
}

Asif Mehmood, Jeff Clark, Mark Brickhouse, Haiwen Chen, Michael McGurr

Asif Mehmood, Jeff Clark, Mark Brickhouse, Haiwen Chen, Michael McGurr, "Hyperspectral chemical agent standoff detection using sparse representation (Withdrawal Notice)," Proc. SPIE 9461, Radar Sensor Technology XIX; and Active and Passive Signatures VI, 94611I (21 May 2015); doi: 10.1117/12.2176242

SPIE. Event: SPIE Defense + Security, 2015, Baltimore, MD, United States 
Hyperspectral chemical agent standoff detection using sparse representation (Withdrawal Notice)

Publisher's Note: This paper, originally published on 21 May 2015, was withdrawn per author request, if you have any questions please contact SPIE Digital Library Customer Service for assistance.

Radar Sensor Technology XIX; and Active and Passive Signatures VI, edited by K. Ranney, A. Doerry,

G. C. Gilbreath, C. T. Hawley, Proc. of SPIE Vol. 9461, 94611I · (c) 2015 SPIE

CCC code: $0277-786 \mathrm{X} / 15 / \$ 18 \cdot$ doi: $10.1117 / 12.2176242$

Proc. of SPIE Vol. $946194611 \mathrm{l}-1$

Downloaded From: https://www.spiedigitallibrary.org/conference-proceedings-of-spie on 26 Apr 2023 Terms of Use: https://www.spiedigitallibrary.org/terms-of-use 